\title{
Difference in rhizosphere soil bacterial community was among six Cinnamomum camphora chemotypes
}

Xiao Zufei, Zhang Beihong, Wang Yangbo, Jin Zhinong, Li Feng*, Peng Yi, Jiang Rui Nanchang Institute of Technology, Jiangxi Provincial Engineering Research Center of Seed-Breeding and Utilization of Camphor Trees, Nanchang, Jiangxi 330099, China

\begin{abstract}
Plant types and soil bacterial communities had a close relationship, understanding the profound association between them contributes to better learn bacterial ecological function for plant growth. In this study, rhizosphere soil of six different chemotype Cinnamomum camphora trees were collected, including $C$. bodinieri var. citralifera, [C. camphora (Linn.) Presl], camphora-type, cineoletype, linalool-type and isoborneol-type. Soil properties content and bacterial communities were analyzed. Two chemotype C. camphora, including [C. camphora (Linn.) Presl] and linalool-type, shaped similar bacterial community structure, decreased Firmcutes relative abundance. richness estimators (Chao1 index and Ace index) of [C. camphora (Linn.) Presl] were decreased compared with the others. Furthermore, soil bacterial community structure was also similar among bodinieri var. citralifera, camphora-type, cineole-type and isoborneol-type. Hence, different chemotype C. camphora altered soil nutrient and shaped rhizosphere bacterial communities.
\end{abstract}

\footnotetext{
* Corresponding author: Li Feng, E-mail address: 840676551@qq.com.
} 
Keywords: Cinnamomum camphora; chemotype; soil nutrient; soil bacterial community diversity and structure

\section{Introduction}

Cinnamomum camphora (L.) Presl is an evergreen tree originating from the south of the Yangtze River in China, has been introduced to other countries [1]. At present, it mainly habitat was tropical and subtropical of Asia, particularly in China, Japan, Korea, and Vietnam [2]. C. camphora is not only used for wood processing and interior decoration of buildings, but has extremely medicinal value and high economic effects [3]. For instance, its wood is usually for furniture making, and it also has been commonly regarded as traditional Chinese herb medicines for the treatment of inflammation-related diseases such as rheumatism, bronchitis and indigestion $[4,5]$. Furthermore, highly qualified essential oil is richness in roots, stems, leaves and wood of C. camphora, such as mint, camphor and patchouli $[6,7]$. Therefore, C. camphora is classified different chemotypes according to chemical composition extracted from it, such as camphora-type and cineole-type [1,8]. Chemotypes are organisms categorized under the same species, subspecies or varieties, with differences in the composition of secondary metabolites [9]. Although, many literatures demonstrate that no obviously difference is in morphology among different $C$. camphora chemotypes, their physiological structure and root secretions are obviously difference $[1,10]$. This result drives potentially changes of surrounding environment growing of C. camphora.

Soil bacteria play a vital role in the terrestrial forest ecosystem, they always gather together on plant root surface to mineralize soil nutrient, decompose litters and 
accelerate nutrient cycle for plant absorption and utilization [11]. However, soil bacterial community structure and diversity are significantly affected by climate, soil texture, vegetation and land use patterns, because they are very sensitive to the changes in surrounding environment [12]. Therefore, soil bacteria are considered as sensitive indicators of soil ecosystem changes. Previous studies suggest that soil bacteria community structure in rhizosphere soil of different plant types also is differences, even under the same site condition [13]. Literatures found that endophytic bacteria in leaves and stems of are differences due to various chemical composition in leaves and stems of different chemotypes $C$. camphora $[3,14]$. However, many studies only focus on the endophytic bacteria and chemical composition of different chemotypes C. camphora, but usually pay little attention on the distribution of bacterial community diversity and structure in its growth.

Based on above, we investigated and analyzed rhizosphere soil properties and bacterial community diversity and structure of six different chemotypes C. camphora, and the deep connection between them. This paper links soil properties and rhizosphere bacterial community among all six chemotypes $C$. camphora to analyze their habitat. The objects of this study were to compare rhizosphere soil bacteria community diversity and structure of six chemotypes C. camphora. This result will not only provide some insights on rhizosphere microecology of different chemotypes C. camphora, also offers some new ideas for the sustainable development of C. camphora.

\section{Materials and methods}

\subsection{Experimental site}


The experimental site is situated in the biotechnology experimental base $\left(115^{\circ} 59^{\prime} 38.40^{\prime \prime}\right.$ E, $\left.28^{\circ} 45^{\prime} 29.20^{\prime \prime} \mathrm{N}\right)$ of Nanchang Institute of Technology, Nanchang City, China. It belongs to subtropical humid monsoon climate, with mild and humid climate, abundant sunshine, abundant rainfall, south wind in summer, north wind in winter, long frost-free period and short freezing period. The mean annual temperature and annual precipitation are $20.5^{\circ} \mathrm{C}$ and $1567 \mathrm{~mm}$, respectively.

\subsection{Experimental treatment and samplings}

Cuttings were from new branches of 3-years-old and different chemotype camphora trees, which include C. bodinieri var. citralifera (NHZ), [C. camphora (Linn.) Presl] (NMZ), camphora-type (NZ), cineole-type (AZ), linalool-type (FZ), isoborneol-type (ZZ). These cuttings were cut into $30 \mathrm{~cm}$ segments with the average ground diameter at $2.0 \mathrm{~cm}$ and soaked in sterile water for $15 \mathrm{~min}$ and then for cutting culture. The trial area was about $198 \mathrm{~m}^{2}(36 \mathrm{~m} \times 6 \mathrm{~m})$, and it was divided into 6 plots with the same size, each plot was $2 \mathrm{~m}$ apart. All cutting were cut into the soil with Row spacing and plant spacing $30 \mathrm{~cm}$, respectively. Approximately $100 \mathrm{~g}$ compound fertilizers were applied into surface soil as base fertilizer in that winter, and $150 \mathrm{~g}$ urea $(\geq 30 \% \mathrm{~N})$ were also average added to each plot in May next year. After cultivating, soil samples (depth of 0-20 cm) of different chemotype cuttings rhizosphere soil were collected after removing surface litters in July 2019. The collected test soil was passed a $2 \mathrm{~mm}$ sieve to remove gravel and roots before divided into two parts. One part was to analysis soil basic physical and chemical properties, and the other soil was storage at $20{ }^{\circ} \mathrm{C}$ for determining bacterial community diversity and structure. 


\subsection{Determinations of soil physicochemical properties}

All soil properties were determined according to Kong [15]. Briefly, dried soil and $25 \mathrm{~mL}$ deionized water were mixed into $50 \mathrm{~mL}$ plastic pipe with ration of 1: 2.5 (soil: water) and shaken for $30 \mathrm{~min}$, the $\mathrm{pH}$ value of the sample was measured using a $\mathrm{pH}$ meter (Sanxin, Shanghai, China). Soil samples were digested with chloric acid and sulfuric acid and then soil total phosphorus (TP) was quantified by the Discrete Auto Analyzer (SmartChem, Brookfield, USA). Soil available phosphorus (AP) content was determined at $700 \mathrm{~nm}$ absorbance with a spectrophotometer by the molybdenum resistance colorimetry method. Soil total $\mathrm{N}$ contents were analyzed with the Discrete Auto Analyzer (SmartChem, Brookfield, USA). Soil ammonium $\left(\mathrm{NH}_{4}{ }^{+}-\mathrm{N}\right)$ and nitrate $\left(\mathrm{NO}_{3}{ }^{-}-\mathrm{N}\right)$ contents were extracted with $2 \mathrm{~mol} \mathrm{~L}^{-1} \mathrm{KCl}$ solution, and they were quantified by the Discrete Auto Analyzer (SmartChem, Brookfield, USA). Soil total potassium (TK) and available K (AP) contents were measured using the Flame Photometer (F-100, Shanghai, China). Soil organic matter (SOM) content was analyzed using the titrating method and then converted into SOM content. All treatments were repeat in ten times.

\section{4 Soil DNA extraction}

Soil total DNA was extracted with a Fast DNA SPIN Kit for Soil (MP Biomedicals, USA) according to the manufacturer protocol. The quantity and quality of extracted DNA were measured using the NanoDrop NC2000 spectrophotometer (Thermo Fisher Scientific, Waltham, MA, USA) and 1.8\% agarose gel electrophoresis, respectively. The extracted DNA was stored at $-20{ }^{\circ} \mathrm{C}$ before analyzing bacterial components.

\subsection{Illumina-MiSeq sequencing and data analysis}


The V3-V4 regions of soil bacterial 16sRNA gene were amplified with common primers 4338F/806R [16], and PCR products were sequenced on the Illumina Miseq platform (Nonogene, China). Raw reads were filtered to obtain high-quality reads with the QIIME software according to the default value. The high-quality reads were classified into operational taxonomic units (OTUs) at 97\% sequence similarity level, and which also was used to calculate soil bacterial Shannon and Simpson indices and Ace and Chaol richness estimators. Meanwhile, bacterial taxonomies were confirmed using the OTUs by the SILVA database (http://www.arb-silva.de).

\subsection{Statistic analysis}

One-way ANOVA was used to analyzed soil basic properties (including soil $\mathrm{pH}$, TP, TN, TK, AP, AK, $\mathrm{NH}_{4}{ }^{+}-\mathrm{N}, \mathrm{NO}_{3}{ }^{-}-\mathrm{N}$ and $\mathrm{SOM}$ content $)$ by the Duncan test $(P<0.05)$ with SPSS 24.0 software (IBM SPSS Inc., USA). Soil bacterial diversity indices (Shannon and Simpson indices) and richness estimators (Ace and Chaol richness estimators) were also performed by One-way ANOVA. The principal co-ordinates analysis was used to reveal soil bacterial community structure. The relationship between soil properties and bacterial relative abundance were performed in RDA. Bacterial biomarkers of different chemotype C. camphora trees rhizosphere soil samples were analyzed using the linear discriminant analysis effect size (LEfSe) method (http://huttenhower.sph.harvard.edu/galaxy/root). The principal co-ordinates analysis was used to reveal soil bacterial community structure. The relationship between soil properties and bacterial relative abundance were performed in RDA. The LEfSe, PCoA, RDA and LDA were performed with the R Project (https://www.r- 
project.org/).

\section{Results}

\section{1 soil properties}

There was no not statistical difference in $\mathrm{pH}$ value among all Camphor trees of different chemotypes (Table 1). At end of this experiment, average SOM content was significant higher at FZ, NZ ZZ and AZ than that of NMZ and NHZ. The highest TP content was $1.22 \pm 0.11$ at $\mathrm{FZ}$ among all soil samples, and AP content was significantly decreased at FZ and NHZ than NMZ, NZ and AZ. Soil K content was the highest at NHZ compared other C. camphor of chemotypes, and AK content was higher at NZ, ZZ and AZ than FZ, NMZ and NHZ. Soil TN content was lower at NMZ and NHZ than that of other Camphor types. For $\mathrm{NH}_{4}{ }^{+}-\mathrm{N}$ content, which was significantly enhanced at $\mathrm{ZZ}$ and FZ, and the highest content was $\mathrm{ZZ}$ at $41.16 \pm 4.59$, the rank of soil sample $\mathrm{NH}_{4}{ }^{+}-\mathrm{N}$ content from high to bottom was $\mathrm{ZZ}>\mathrm{FZ}>\mathrm{NMZ}>\mathrm{AX}>\mathrm{NHZ}>\mathrm{NZ}$. Soil $\mathrm{NO}_{3}{ }^{-}-\mathrm{N}$ content was higher at $\mathrm{ZZ}, \mathrm{AZ}, \mathrm{FZ}$ and $\mathrm{NZ}$ than $\mathrm{NMZ}$ and $\mathrm{NHZ}$.

\subsection{Soil bacterial community diversity and sequence data}

The similarities and differences of OTUs among the rhizosphere soil samples of different chemotype C. camphor trees were demonstrated in a six-set Venn diagram (Figure 1). The six different chemotype C. camphor trees shared 2287 OTUs, and the unique OTUs were 379, 371, 212, 238, 446 and 237 for the AZ, FZ, NZ, NHZ, NMZ and $\mathrm{ZZ}$ rhizosphere soils, respectively.

Soil bacterial Shannon index was lower at NMZ than that of NHZ. Shannon index at NMZ was lower than NHZ (Figure 1a). There was no significantly difference in 
Simpson index among all chemotype C. camphor trees (Figure 2b). Surprisingly, bacterial richness estimators (Chao1 index and Ace index) were decreased at NMZ, and they were highest at $\mathrm{AZ}(3423.81 \pm 361.33,3445 \pm 333.56)$ among all Camphor trees of different chemotype (Figure 2c and 2c).

\subsection{Soil bacterial community structure}

Based on the SILVA database, soil bacterial OTUs were assigned into predominant phyla as following: Proteobacteria, Actinobacteria, Acidobacteria, Bacteroidetes, Chloroflexi, Firmicutes, Gemmationadetes, Latescibacteria and Rokubacteria. At phylum taxa level (Figure 3), Proteobacteria, Acidobacteria, Bacteroidetes, Chloroflexi, Firmicutes, Gemmationadetes and Latescibacteria relative abundances were significantly altered at rhizosphere soil of different chemotype $C$. camphor trees, suggesting that soil bacteria were reassembled at different chemotype $C$. camphor trees. The relative abundance of Proteobacteria was lower at NMZ than their counterparties at $\mathrm{ZN}$ and $\mathrm{AZ}$ (Figure 4a). Soil Actinobacteria relative abundance significantly increased at FZ, NMZ and NHZ relative to NZ and AZ (Figure 4b). For Bacteroidetes, its relative abundance was higher at NMZ and NHZ than that at NZ and ZZ (Figure 4c), and there was a significant difference in Chloroflexi relative abundance between NMZ and AZ (Figure 4d). While, Firmicutes relative abundance at FZ and NMZ decreased compared with other counterparties (Figure 4e). The relative abundance of Gemmationadetes enhanced among ZZ, AZ and ZN relative to NMZ and NHZ (Figure 4f). Compared with both NZ and AZ, Latescibacteria relative abundance was higher at FZ (Figure 4g). 


\section{3 bacterial community structure}

The LefSe analysis indicated that there were statistically significant associations among predominant bacterial taxa in rhizosphere soil of different chemotype $C$. camphora trees. The phylum Acidobacteria was stimulated by the root exudations of FZ, MHZ and NMZ, suggesting that these species may potentially be recruited by them and as their biomarkers for rhizosphere soil (Figure 5).

The two-dimensional PCoA plot explained $36.33 \%$ of the total variation, and the PCoA1 accounted for $23.77 \%$ of the total variance (Figure 6). The samples both of NMZ and FZ was highly similar and they mainly distributed negatively along with PCoA1 axis. Furthermore, the others samples were also extreme similar with each other, and even $95 \%$ confidence ellipse of ZZ included NHZ and AZ, and this result confirmed that soil bacterial community structure was distinct divided two parts.

\subsection{Linking soil properties and bacterial community}

The RDA analysis revealed the comprehensive relationship between soil properties and dominate bacterial taxa (Figure 7). A positive associate was shown between $\mathrm{pH}$, the relative abundance among Actinobacteria, Acidobacteria, Bacteroidetes and Latescibacteria, but which had negative with $\mathrm{SOM}, \mathrm{TN}$ and $\mathrm{NO}_{3}{ }^{-}-\mathrm{N}$ content. In addition to, Proteobacteria relative abundance was negatively with TP, TK and $\mathrm{NH}_{4}{ }^{+}-\mathrm{N}$ content.

\section{Discussion}

\subsection{Comparison bacterial community diversity}

At the end of this study, Chaol and Ace richness estimators of NMZ were 
significantly decreased compared with other five chemotypes C. camphora. This result suggested that bacteria community diversity was lower than other chemotypes $C$. camphora, this was also confirmed in fig that the relative abundance of Firmicutes and Gemmationadetes was the lowest. We predicted that the life activity of bacteria may be impacted by soil SOM concentration. SOM coming from litters and microbial residuals is commonly considered as carbon by bacteria, and which can provide substantial and persistent energy for bacterial life activity $[17,18]$. In this study, high relative abundance of Acidobacteria had negative associate with SOM content, this also can account for the decreasing of SOM content of NMZ, due to the Acidobacteria could degrade organic matters from litters, maintaining soil available nutrient supply [19, 20].

Apart from the effects of SOM content on soil bacterial community diversity, mineral nutrient also plays an important role on composition of bacteria [21]. At our recent study, soil bacterial relative abundance was significantly altered in rhizosphere soil of different chemotypes C. camphora and which shaped different bacterial communities. We predicted that root exudation may be the main driver factor. Root secretions coming from different chemotypes C. camphora, might be difference, even had one more distinct difference $[1,22,23]$. Some bacteria preferencing this distinct substance are recruited and appealed to rich on plant rhizosphere, but soil bacteria may also be inhibited indirectly by root secretions due to fiercely competition for nutrients and spaces was among bacteria $[24,25]$. Therefore, nutrient supply was also another major influence factor. For example, Proteobacteria phylum participates in $\mathrm{N}$ transformation processes and accelerates $\mathrm{N}$ cycle, they also can efficiently convert and 
utilize root exudation, in particular in transforming the low molecular weight substance [26]. In this paper, Proteobacteria phylum was richness on rhizosphere soil, this may be caused that 1) Proteobacteria was attracted and recruited to transform $\mathrm{N}$ for development and growth of C. camphora under $\mathrm{N}$ addition condition [27]; 2) Proteobacteria phylum may also be recruited by root exudation due to they were the major phylum convert and utilize root exudation [28].

\subsection{Comparison bacterial community structure}

There is a close relationship between vegetation composition and soil physical and chemical properties. Plant affects soil physical and chemical properties through litterfall and root exudates, thus affecting the composition and structure of soil bacterial communities [11]. Previous studies indicated that changes in plant species had a significant effect on bacterial community structure [11]. Therefore, it can be better understood the impact of vegetation types on soil microbial ecosystems and function by studying the relationship between soil microorganisms and vegetation.

In this study, the PCoA analysis confirmed that soil bacterial community structure was divided obviously two parts among six different chemotypes C. camphora, one part was FZ and NMZ, and another was AZ, ZZ, NZ and NHZ. This result suggested that rhizosphere of six different chemotypes $C$. camphora trees were mainly divided two forms. It can be explained that there was something similar between root exudations of FZ and NMZ, but which was different form root exudations that secreting by other four different chemotype $C$. camphora trees. In addition, these similar root exudations recruited countless bacteria to gather in the rhizosphere and shaped the 
bacterial community structure. Previous study also found that plant genotype was one of the dominate factors affecting rhizosphere soil bacterial community structure. Such as the microbiome community of rhizosphere of wheat and chickpea was significant difference due to their root drive the composition and function of the rhizosphere microorganisms [29]. In return, changes in composition and function of the rhizosphere microorganisms can alter soil nutrients then leading to redistribution of soil microbe [30]. Besides, rhizosphere bacterial community structure was also difference at the period of plant growth due to changing plant root architecture and nutrient supply [31]. Hence, difference in bacterial community structure was among six different chemotypes C. camphora.

\section{Conclusion}

The Illumina-MiSeq sequencing analysis suggested that rhizosphere soil bacterial community diversity and structure of six different chemotype C. camphora trees were significant difference, the relative abundance of phylum Firmicutes at FZ and NMZ decreased compared the others, but Acidobacteria relative abundance of FZ and NMZ was higher than NZ, ZZ and AZ. Moreover, soil bacterial community structure was highly similar between FZ and NMZ, as well as among ZZ, NZ, AZ and NHZ. SOM content had reduced trend at $\mathrm{NHZ}$ and $\mathrm{NMZ}$, and soil $\mathrm{pH}$ was no significant difference among all different chemotype C. camphora trees. In conclusion, different chemotype C. camphora trees altered soil properties and shaped different rhizosphere soil bacterial communities, and NMZ and FZ had highly similar aspect on bacterial community structure. Therefore, different chemotype C. camphora trees had different rhizosphere 
bacterial communities.

\section{Declaration of Competing Interest}

The authors declare that they have no known competing financial interests or personal relationships that could have appeared to influence the work reported in this paper.

\section{Acknowledgments}

This study was financially supported by the Forestry Science and Technology Innovation Project of Jiangxi Provincial Department Forestry, China (201904), the Key Research and Development Project of Science and Technology Department of Jiangxi Province, China (20192BBFL60012), Research Specific Project of Camphora Tree of Jiangxi Provincial Department Forestry, China (202007), Major Research and Development Specific Project of Science and Technology Department of Jiangxi Province, China(20203ABC28W016).

\section{References}

1. Guo, X.; Cui, M.; Deng, M; Liu. X.; Huang. X; Zhang, X. Molecular differentiation of five Cinnamomum camphora chemotypes using desorption atmospheric pressure chemical ionization mass spectrometry of raw leaves. Sci. Rep. 2017, 7, 46579.

2. Meng, J.; Li, M.; Guo, J.; Zhao, D.; Tao, J. Predicting suitable environments and potential occurrences for Cinnamomum camphora (Linn.) Presl. Forests 2021, 12, 1126. 
3. Singh, R.; Jawaid, T. Cinnamomum camphora (Kapur): Review. Pharmacogn. J. $2012,4,1-5$.

4. Lee, H.J.; Hyun, E.; Yoon, WJ.; Kim, BH.; Rhee, MH.; Kang, H.K. In vitro antiinflammatory and anti-oxidative effects of Cinnamomum camphora extracts. J. Ethnopharmacol. 2006, 103, 208-216.

5. Xiao, S., Yu, H.; Xie, Y. The anti-inflammatory potential of Cinnamomum camphora (L.) J. Presl essential oil in vitro and in vivo. J. Ethnopharmacol. 2020, $267,113516$.

6. Yang, F.; Long, E.; Wen, J.; Cao, L.; Zhu C.; Hu, H. Linalool, derived from Cinnamomum camphora (L.) Presl leaf extracts, possesses molluscicidal activity against Oncomelania hupensis and inhibits infection of Schistosoma japonicum. Parasit Vectors. 2014, 7, 407.

7. Chen, S.; Zheng, T.; Ye, C. Algicidal properties of extracts from Cinnamomum camphora fresh leaves and their main compounds. Ecotoxicol. Environ. Saf. 2018, $163,594-603$.

8. Wu, C.; Chang, S.; Tung, C.; Hou, C.; Gogorcena, Y.; Chu, F. Identification of hybridization and introgression between Cinnamomum kanehirae Hayata and $C$. camphora (L.) Presl using genotyping-by-sequencing. Sci. Rep. 2020, 10, 15995.

9. Desjardins A.E. Natural product chemistry meets genetics: When is a genotype a chemotype? J. Agr. Food Chem. 2008, 56, 7587-7592.

10. Satyal, P.; Jones, T.; Lopez, E.; McFeeters, R.; Ali, N.; Mansi I. Chemotypic characterization and biological ctivity of Rosmarinus officinalis. Foods 2017, 6, 20. 
11. Singh, J.; Raghubanshi, A.; Singh. R.; Srivastava. S. Microbial biomass acts as a source of plant nutrients in dry tropical forest and savanna. Nature 1989, 338, 499500.

12. Jiang, Y.; Luan, L.; Hu, K.; Liu, M.; Chen, Z.; Geisen, S. Trophic interactions as determinants of the arbuscular mycorrhizal fungal community with cascading plant-promoting consequences. Microbiome 2020, 8, 142.

13. Zhang, X.; Gagnon, B.; Ziadi, N.; Cambouris, A.N.; Alotaibi. K.D.; Hu, Z. Soil phosphorus fractionation as affected by paper mill biosolids applied to soils of contrasting properties. Front. Environ. Sci. 2020, 23, 8.

14. Zhang G., Huang Q., Bi X., Liu Y., Yuan Z. Analysis of endophytic bacterial community diversity and metabolic correlation in Cinnamomum camphora. Arch. Microbiol. 2020, 202,181-189.

15. Kong. J.; Yang. J.; Bai. E. Long-term effects of wildfire on available soil nutrient composition and stoichiometry in a Chinese boreal forest. Sci. Total Environ. 2018, $642,1353-1361$.

16. Wang, Y.; Ji, H.; Hu, Y.; Wang, R.; Rui, J.; Guo, S. Different selectivity in fungal communities Between manure and mineral fertilizers: A study in an alkaline soil after 30 years fertilization. Front Microbiol 2018, 9, 2613.

17. Zhang, M.; Zhang, W.; Bai, S.H.; Niu, Y.; Hu, D.; Ji, H. Minor increases in Phyllostachys edulis (Moso bamboo) biomass despite evident alterations of soil bacterial community structure after phosphorus fertilization alone: Based on field studies at different altitudes. Forest Ecol. Manag. 2019, 451, 117561. 
18. Ye, Y.; Yue, L. Phosphorus addition decreases microbial residual contribution to soil organic carbon pool in a tropical coastal forest. Glo. Chang. Biol. 2021, 0, 113.

19. Zhang, M., O'Connor, P.J.; Zhang, J.; Ye, X. Linking soil nutrient cycling and microbial community with vegetation cover in riparian zone. Geoderma 2021, 384, 114801.

20. Manna, M.C.; Swarup. A.; Wanjari. RH.; Ravankar, H.N.; Mishra B., Saha, M.N. Long-term effect of fertilizer and manure application on soil organic carbon storage, soil quality and yield sustainability under sub-humid and semi-arid tropical India. Field Crop Res. 2005, 93, 64-80.

21. Wan, W.; Tan, J.; Wang, Y.; Qin, Y.; He, H.; Wu, H. Responses of the rhizosphere bacterial community in acidic crop soil to $\mathrm{pH}$ : Changes in diversity, composition, interaction, and function. Sci. Total Environ. 2020, 700, 134418.

22. Phillips, R.P.; Finzi AC.; Bernhardt, E.S. Enhanced root exudation induces microbial feedbacks to $\mathrm{N}$ cycling in a pine forest under long-term $\mathrm{CO}_{2}$ fumigation. Ecol. Leff. 2011, 14,187-94.

23. Wang, W.; Li, D.; Huang, X.; Yang, H.; Qiu, Z.; Zou, L. Study on antibacterial and quorum-sensing inhibition activities of cinnamomum camphora leaf essential oil. Molecules 2019, 24, 3792.

24. Preece, C.; Peñuelas, J. A return to the wild: Root exudates and food security. Trends Plant Sci. 2020, 25, 14-21.

25. Kang, H.; Gao, H.; Yu, W.; Yi, Y.; Wang, Y.; Ning, M. Changes in soil microbial 
community structure and function after afforestation depend on species and age: Case study in a subtropical alluvial island. Sci. Total Environ. 2018, 625, 1423-32.

26. Campbell, B.J.; Enge,1 A.S.; Porter, M.L.; Takai K. The versatile $\varepsilon$-proteobacteria: key players in sulphidic habitats. Nat. Rev. Microbiol. 2006, 4, 458-68.

27. Moulin, L.; Munive, A. Nodulation of legumes by members of the $\beta$-subclass of Proteobacteria. NATURE. 2002, 411, 948.

28. Song, Y.; Li, X.; Yao, S.; Yang, X.; Jiang, X. Correlations between soil metabolomics and bacterial community structures in the pepper rhizosphere under plastic greenhouse cultivation. Sci. Total Environ. 2020, 728, 138439.

29. Zhou, Y.; Coventry, D.R.; Gupta, V.VS.R.; Fuentes, D.; Merchant, A.; Kaiser, B.N. The preceding root system drives the composition and function of the rhizosphere microbiome. Genome Biol. 2020, 21, 89.

30. Liu, J.; Qi, W.; Li, Q.; Wang, S.; Song, C.; Yuan, X. Exogenous phosphorussolubilizing bacteria changed the rhizosphere microbial community indirectly. Biotech $2020,10,164$.

31. Su, Z.; Xiao.; Q. Shen.; J. Chen, H.; Yan, S.; Huang W. Metabolomics analysis of litchi leaves during floral induction reveals metabolic improvement by stem girdling. Molecules 2021, 26, 4048. 
Table 1. Rhizosphere soil properties of six chemotype Cinnamomum camphora trees. Different lowercase letters represent significant differences, $p<0.05 . \mathrm{NHZ}$, C. bodinieri var. citralifera, NMZ, [C. camphora (Linn.) Presl], NZ, camphora-type, AZ, cineole-type, FZ, linalool-type, ZZ, isoborneol-type.

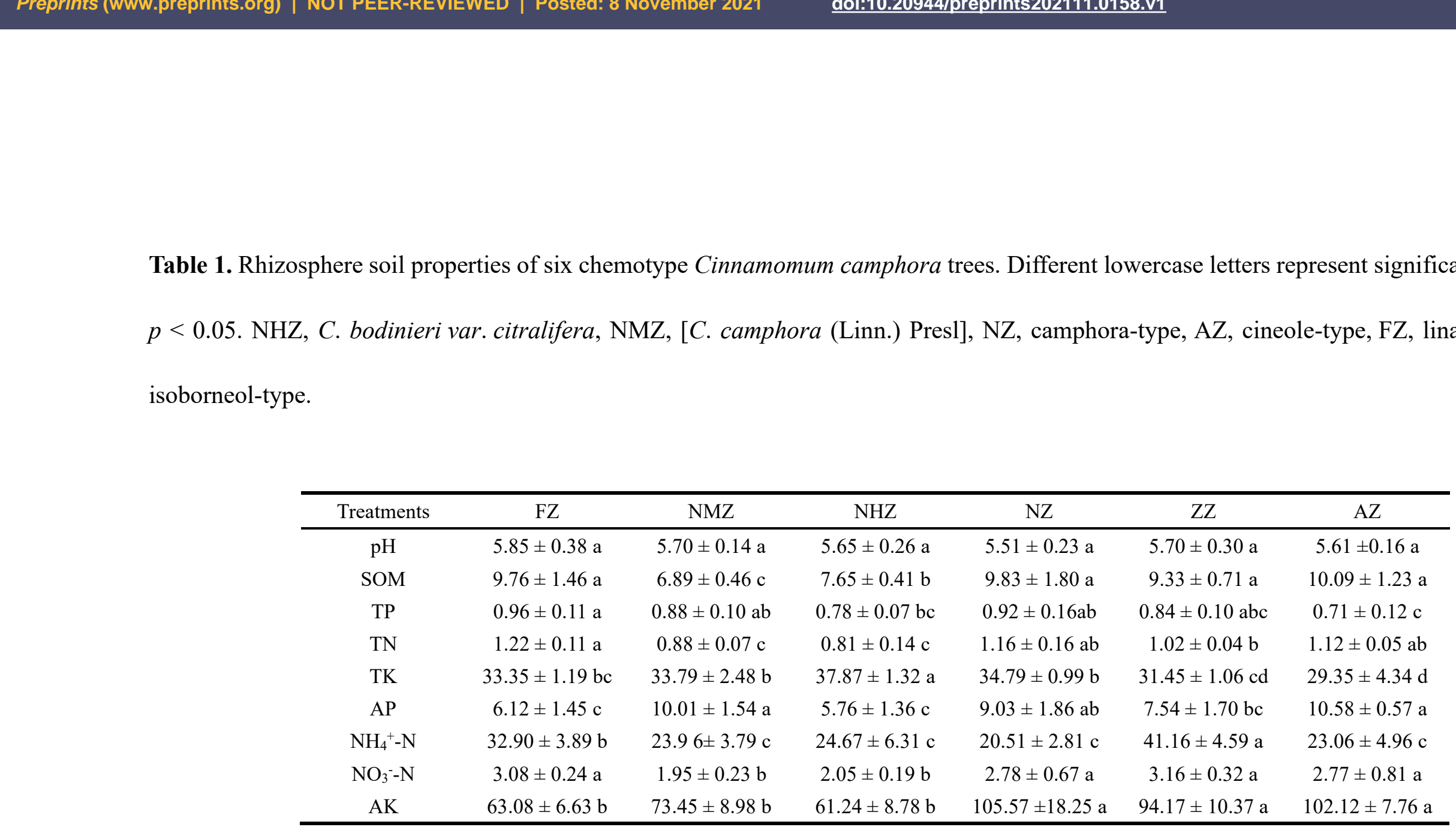



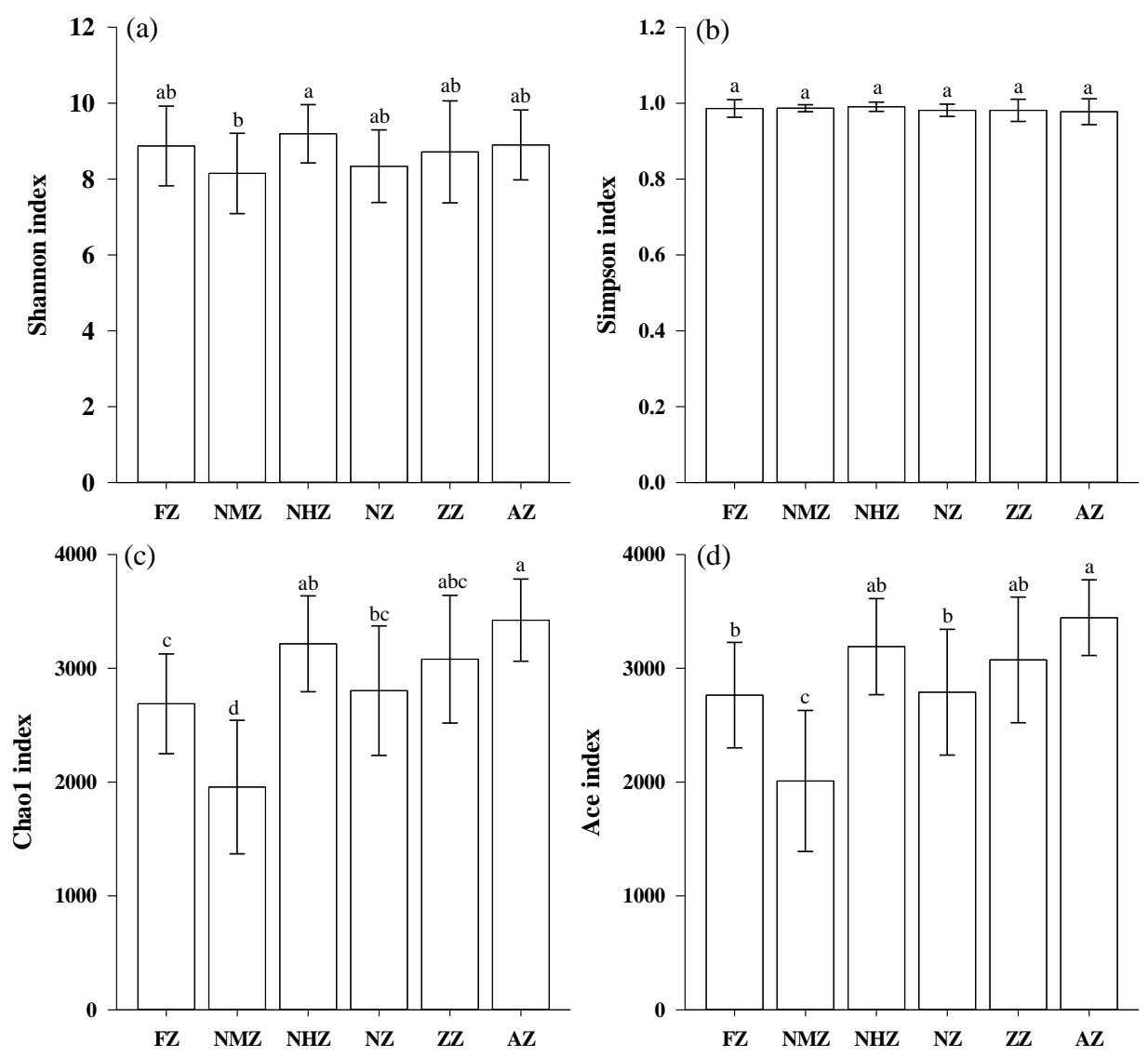

Figure 1. Soil bacterial community diversity of different chemotype C. camphora trees.

(a) bacterial Shannon index, (b) Simpson index, (c) Chaolindex and (d) Ace index.

Different lowercase letters represent significant differences, $p<0.05$. NHZ, $C$. bodinieri var. citralifera, NMZ, [C. camphora (Linn.) Presl], NZ, camphora-type, AZ, cineole-type, FZ, linalool-type, ZZ, isoborneol-type. 


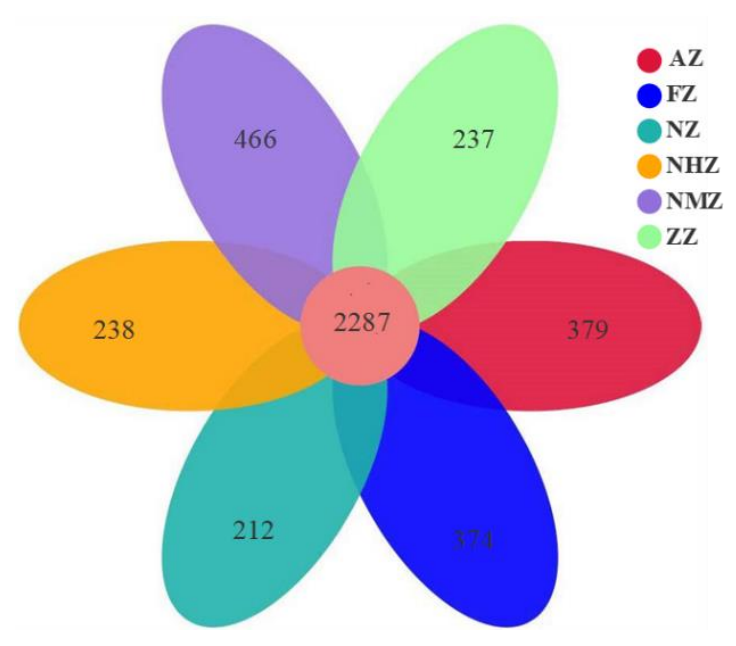

Figure 2. The Venn diagram showing the numbers of unique OTUs of the five treatments. Details are described in Figure 1.

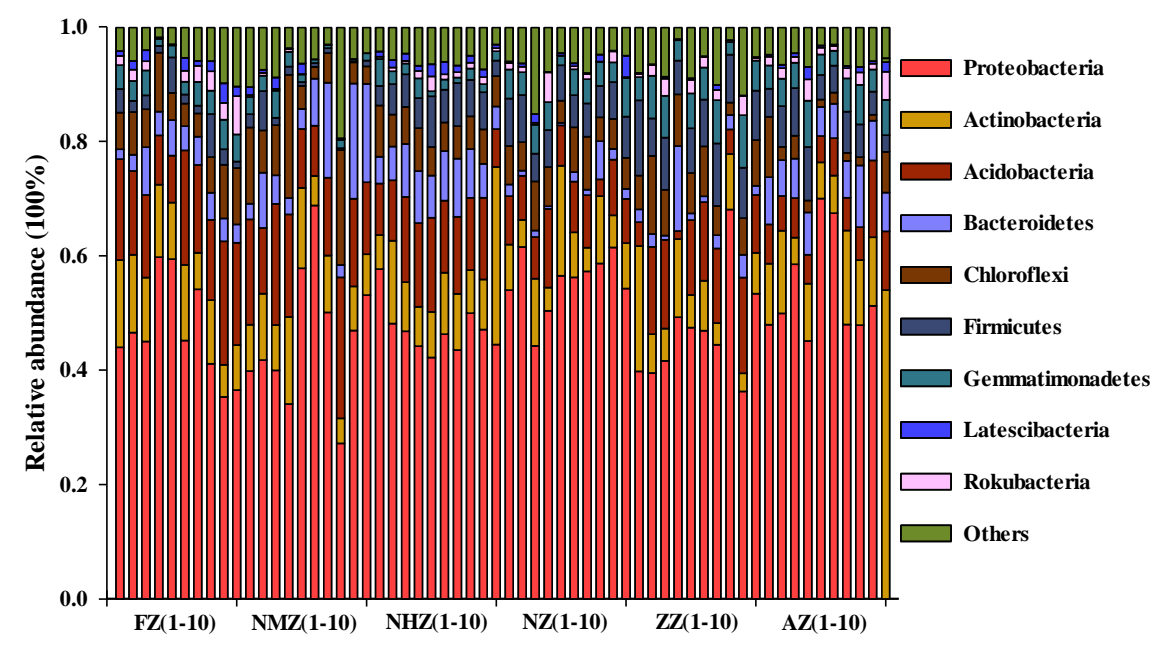

Figure 3. Soil bacterial taxonomic distribution at the phylum level. Details are described in Figure 1. 

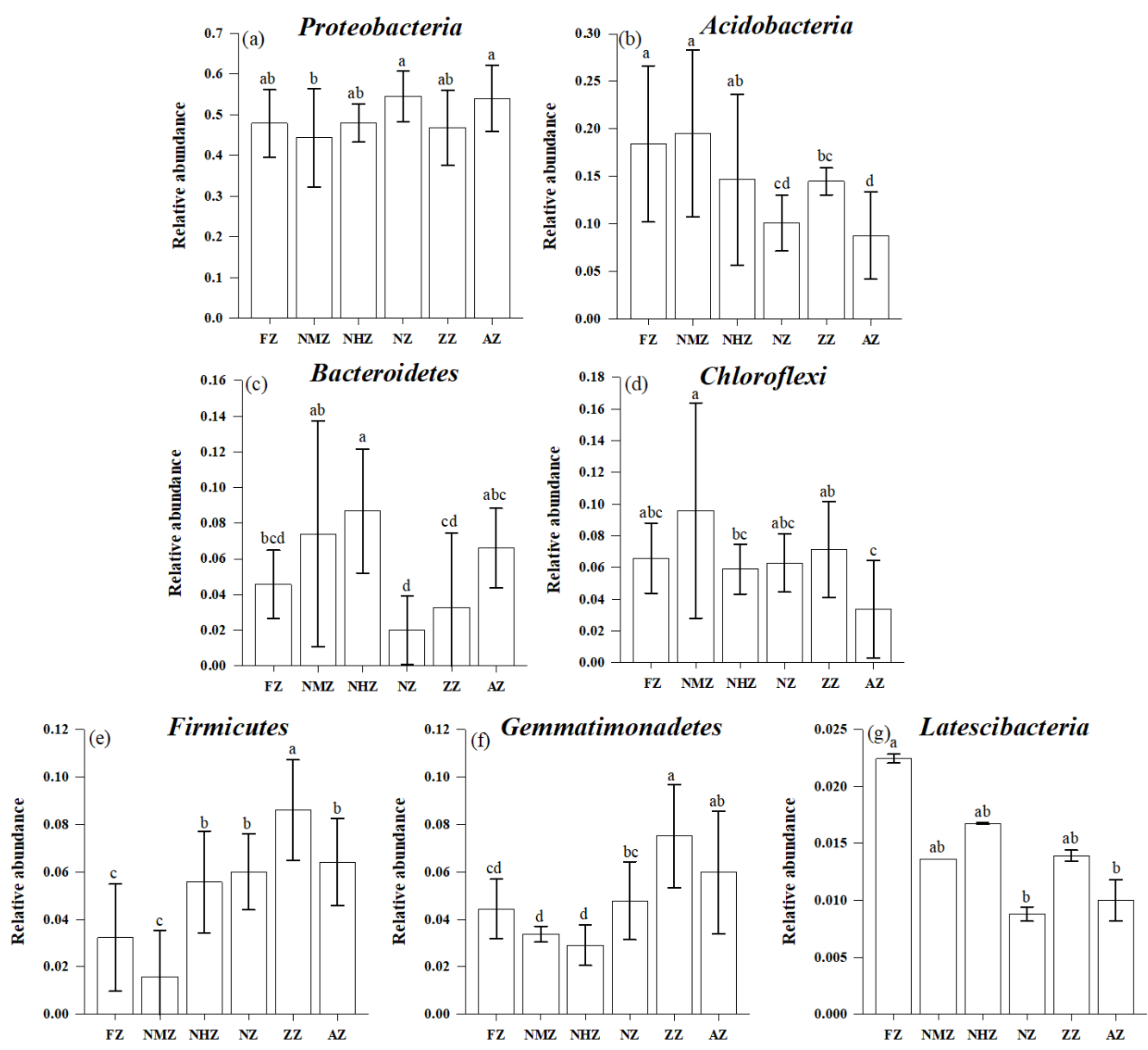

Figure 4. Soil bacterial taxonomic distribution at the phylum level. Details are described in Figure 1. 


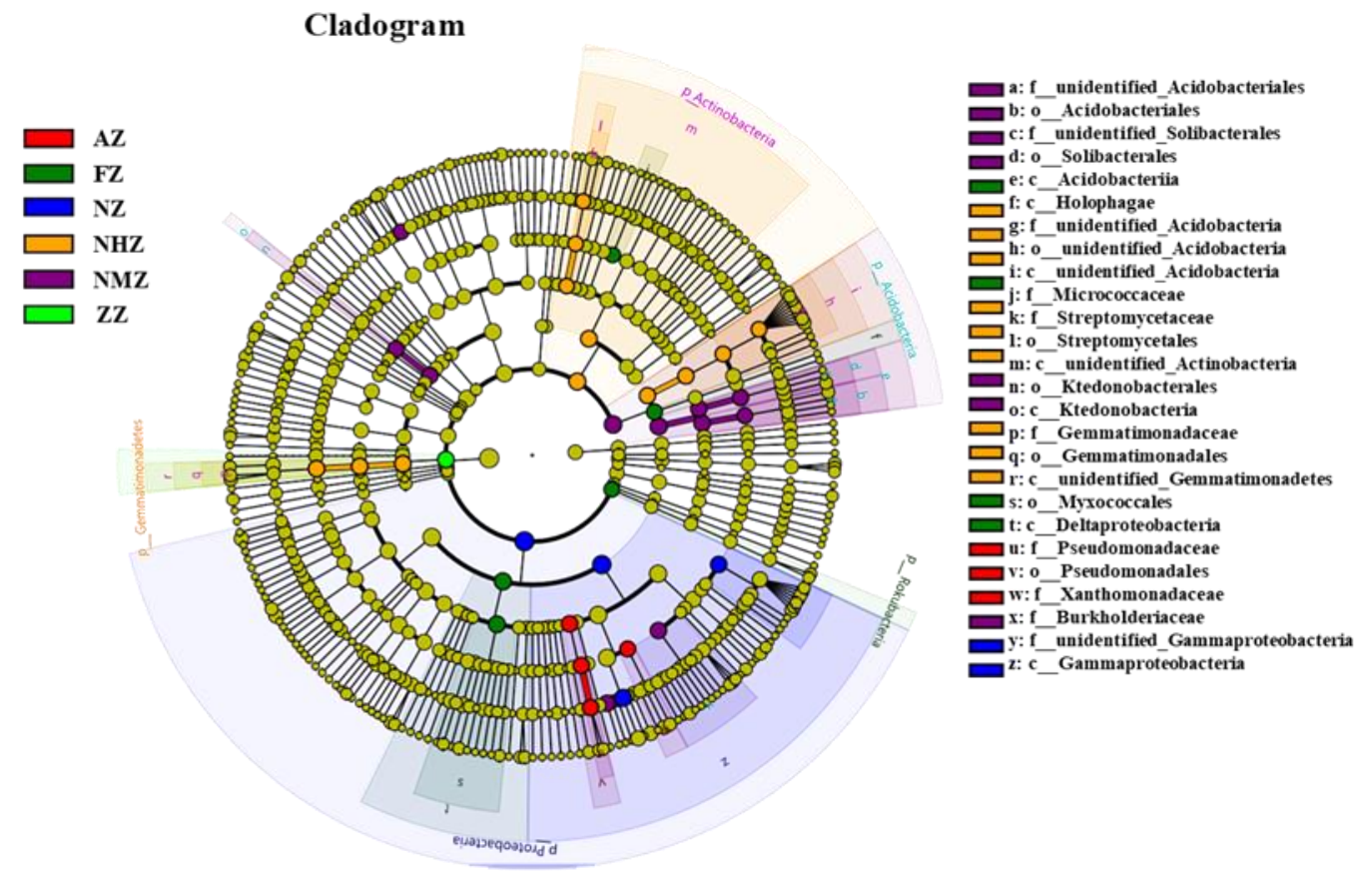

Figure 5. The significantly different abundant taxa of bacteria in the five treatments.

Details are described in Figure 1. 


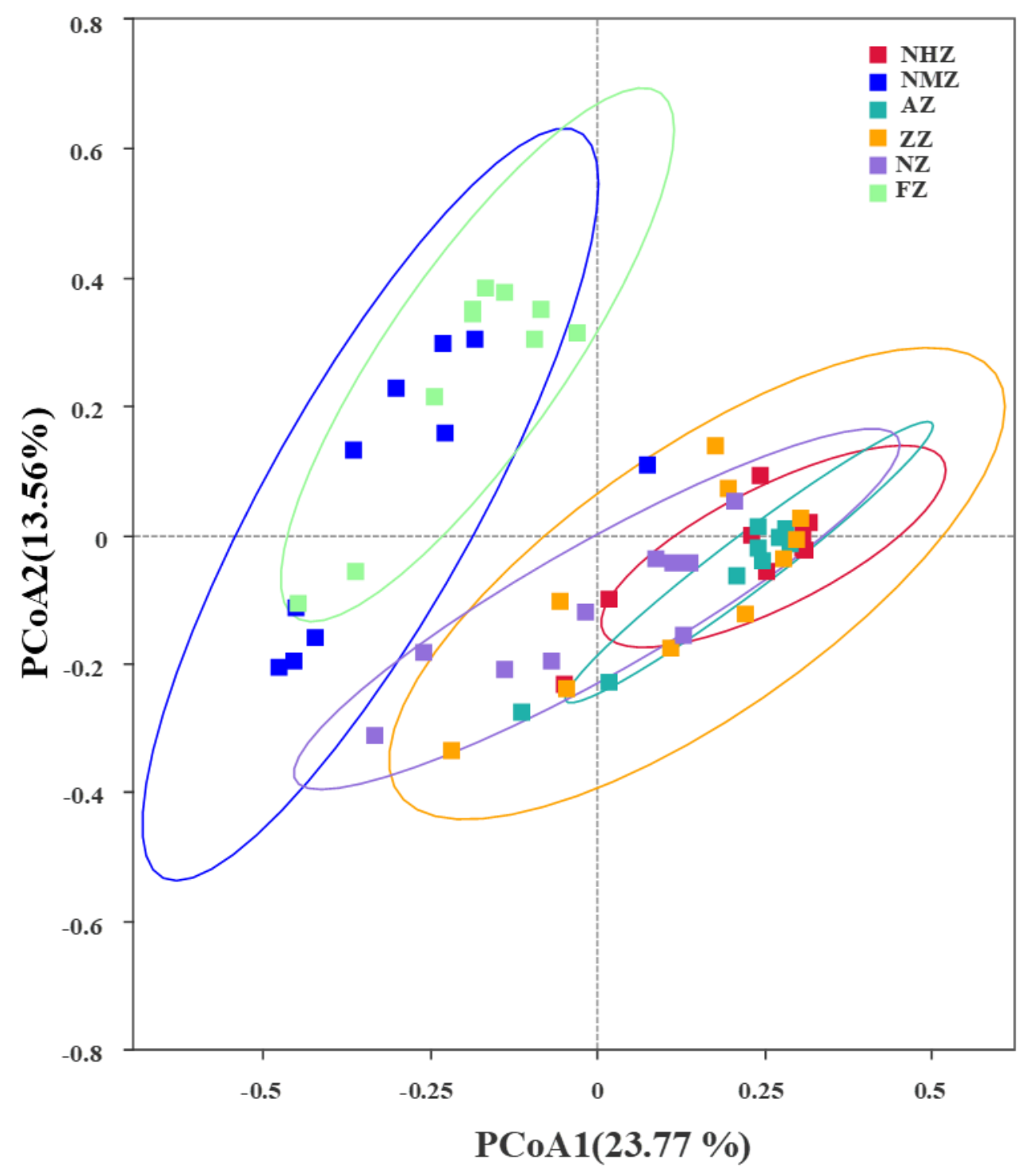

Figure 3. Principal coordinate analysis revealing the entire soil bacterial community structure. Details are described in Figure 1. 


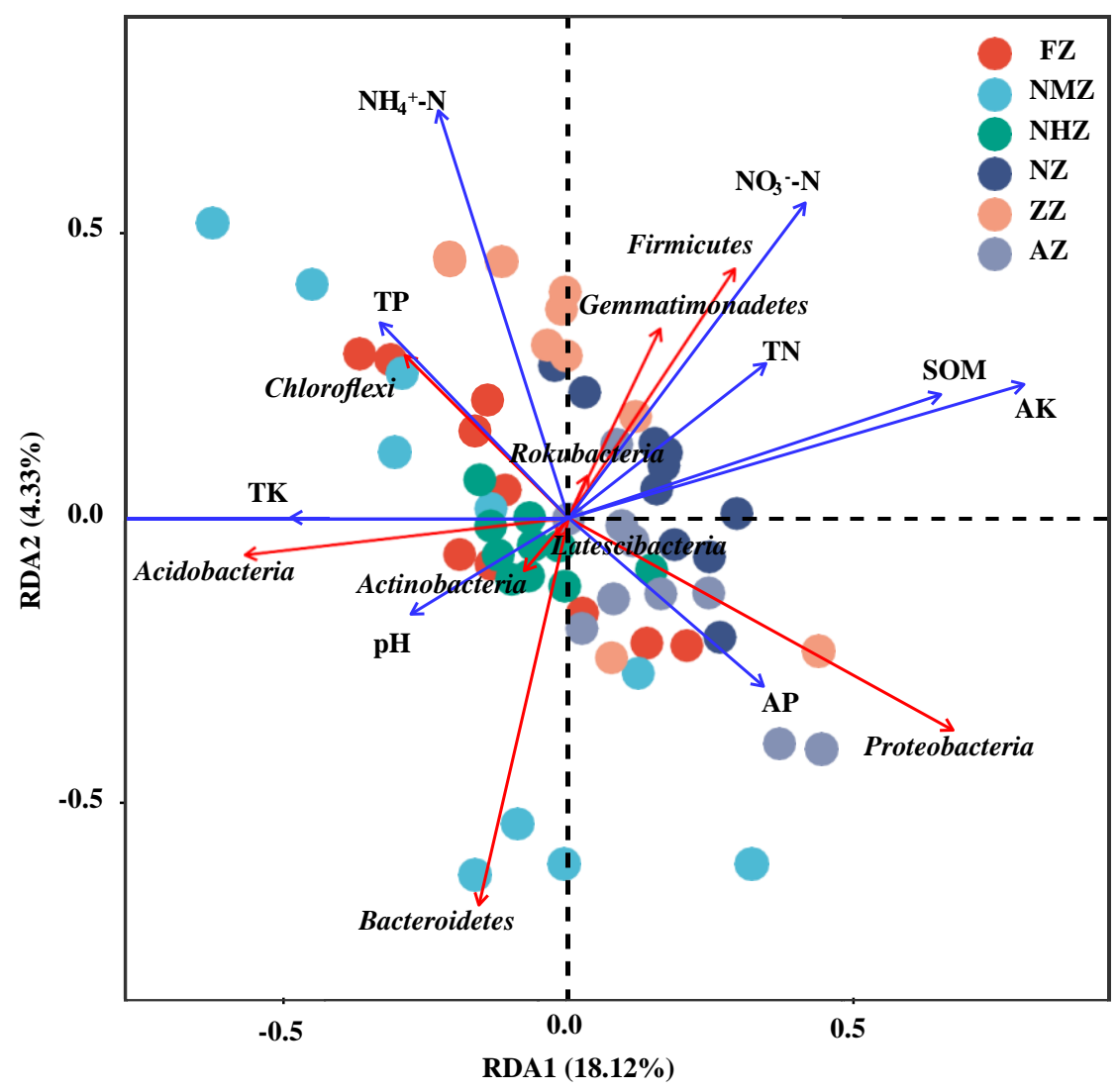

Figure 4. Principal redundance analysis revealing the comprehensive linkages between soil properties and bacterial community. Details are described in Figure 1. 